\title{
Optimization of the Document Placement in the RFID Cabinet
}

\author{
Maciej Kiedrowicz ${ }^{1}$ Tadeusz Nowicki ${ }^{1, a}$, Robert Waszkowski ${ }^{1}$, Zbigniew Wesołowski ${ }^{1}$, Kazimierz Worwa ${ }^{1}$ \\ ${ }^{1}$ The Faculty of Cybernetics, Military University of Technology, 00-908 Warsaw, Kaliskiego 2, Poland
}

\begin{abstract}
The study is devoted to the issue of optimization of the document placement in a single RFID cabinet. It has been assumed that the optimization problem means the reduction of archivization time with respect to the information on all documents with RFID tags. Since the explicit form of the criterion function remains unknown, for the purpose of its approximation, the regression analysis method has been used. The method uses data from a computer simulation of the process of archiving data about documents. To solve the optimization problem, the modified gradient projection method has been used.
\end{abstract}

\section{Introduction}

One of the important problems related to the use of the document archivization system with the RFID (Radiofrequency identification) technology [1,2], is the issue of such placement of the set of documents on particular shelves of the RFID cabinet that minimize the length of the time interval of registering the data about these documents in a database of an information system. To solve this problem, the standard optimization approach may be applied [3, 4]. One of the issues, which need to be solved by using such approach, is the problem of collecting data necessary for approximation of the criterion function. Since the process of data collection is time-consuming and laborious, to improve it, the stochastic simulation method has been applied [5-7]. It is based on generating pseudorandom real numbers, whose values are interpreted as the length of time intervals of archiving the information about the RFID-tagged documents. Thanks to such features as the ability to quickly generate a wide variety of data and flexibility in planning and performing various experiments, computer simulation has become in recent years a kind of modus operandi of many scientific specialties [8]. On the basis of the simulation data, local approximations of the criterion function are performed using the regression analysis method [9-11]. Thanks to applying the complete two-level plan of experiments $[12,13]$, the computational complexity of the estimation process of the local linear parameters of the regression functions was reduced.

Section 2 formulates the optimization problem. Section 3 describes the method for solving the problem. In particular, section 3.1 presents the method for estimation the parameters of the local linear regression functions, whereas section 3.2 describes an algorithm for solving the optimization problem reflecting the modified gradient projection method [14, 15].
Let $(\Omega, \mathcal{F}, P)$ be the probabilistic space, where all random variables are defined. Let us use the following notation: $\mathbb{N}$ - the set of natural numbers, $\mathbb{R}$ - the set of real numbers, $W N\left(0, \sigma^{2}\right)$ - the white noise with zero expected value and variation $\sigma^{2}$.

\section{Formulation of the optimization problem}

Let us assume that the RFID cabinet is composed of $S$, $S>0$, shelves for documents with the RFID tags. Let $\mathcal{S}=\{1, \ldots, S\}$ be a set of shelf numbers. Let $m_{s}, m_{s} \geq 0$ be the number of free spaces for the documents on shelf number $s, s \in \mathcal{S}$. Let $x_{s}, 0 \leq x_{s} \leq m_{s}$ be the number of documents stored on shelf number $s$. We will assume that $D, D>0$, documents must be stored in the cabinet. Let $\mathcal{D}=\{1, \ldots, D\}$ be a set of numbers of such documents. The point is to placement the documents from set $\mathcal{D}$ in such a manner so that minimize the length of time interval of archiving the information about the RFIDtagged documents. Let $y \in \mathbb{R}$ means the length of the time interval.

The optimization issue can be formulated as follows

$$
\mathrm{f}(\hat{\mathbf{x}})=\min _{\mathbf{x} \in \mathcal{X}_{0}} \mathrm{f}(\mathbf{x}),
$$

where:

- $\mathrm{f}: \mathbb{R}^{S} \rightarrow \mathbb{R}$ is the criterion function of unknown explicit form, whose the values are interpreted as the length of time intervals of archiving the information about the RFID-tagged documents from $\operatorname{set} \mathcal{D}$;

- $\mathcal{X}_{0}$ is the set of feasible solutions in the form of

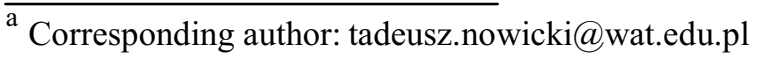




$$
\mathcal{X}_{0}=\left\{\mathbf{x} \in \mathbb{R}^{S}: \mathbf{x} \geq \mathbf{0}, \mathbf{g}(\mathbf{x}) \leq \mathbf{0}, \mathrm{g}_{S+1}(\mathbf{x})=0\right\},
$$

and $\mathbf{g}(\mathbf{x})=\left[\mathrm{g}_{1}(\mathbf{x}), \mathrm{g}_{2}(\mathbf{x}), \ldots, \mathrm{g}_{S}(\mathbf{x})\right]^{T}$ is a vector constraint function, $\mathrm{g}_{s}: \mathbb{R}^{S} \rightarrow \mathbb{R}$ is a constraint function in the form of $\mathrm{g}_{s}(\mathbf{x})=\mathbf{x}^{T} \mathbf{a}_{s}-m_{s}$, $\mathbf{a}_{s}=[0, \ldots, 0, \underset{s}{1}, 0, \ldots, 0]^{T}$, for $s \in \mathcal{S} ; \mathrm{g}_{S+1}: \mathbb{R}^{S} \rightarrow \mathbb{R}$ is a constraint function in the form of $\mathrm{g}_{S+1}(\mathbf{x})=D-\mathbf{x}^{T} \mathbf{1}, \mathbf{1}$ is a unit vector.

Subject 1. If $\sum_{s \in \mathcal{S}} m_{s} \geq D, m_{s} \geq 0, s \in \mathcal{S}, D>0$, then the set of permissible solutions $\mathcal{X}_{0}$ (2) is not empty, closed and convex.

Proof. The set of $\mathcal{X}_{0}$ is closed, since the functions $\mathbf{x}^{T} \mathbf{a}_{s} \leq m_{s}, m_{s} \geq 0, s \in \mathcal{S}$, limit it from the top, and the limitations $\mathbf{x} \geq \mathbf{0}$ and $\mathbf{x}^{T} \mathbf{1} \geq D$ limit this set from the bottom. The set of $\mathcal{X}_{0}$ is not empty, since if $\sum_{s \in \mathcal{S}} m_{s} \geq D$ and $m_{s} \geq 0, s \in \mathcal{S}, D>0$, then the set $\mathcal{X}_{0}$ includes points $\quad \mathbf{x} \geq \mathbf{0}$ meeting the constraint criteria $\mathbf{x}^{T} \mathbf{a}_{s} \leq m_{s}, s \in \mathcal{S}$. The convexity of the set $\mathcal{X}_{0}$ is due to the fact that all limitations are linear.

\section{Method for solving the optimization problem}

Problem (1) is a nonlinear problem of the mathematical programming with linear constraints. If the mapping $\mathrm{f}: \mathbb{R}^{S} \rightarrow \mathbb{R}$ is differentiable and set $\mathcal{X}_{0}$ is closed and convex, the problem may be solved by the modified gradient projection method. This method consists of generating a sequence of approximations:

$$
\mathbf{x}^{k+1}=\mathbf{x}^{k}+\tau_{k} \mathbf{d}^{k}, \quad k=0,1, \ldots,
$$

where: $\mathbf{x}^{0} \in \mathcal{X}_{0}$ is a predefined initial point, $\tau_{k}>0$ is the $k$ th step size, the value of which can be determined by the Armijo rule, $\mathbf{d}^{k} \in \mathbb{R}^{S}$ is the direction, in which the minimum of the criterion function $\mathrm{f}$ is searched. Since the form of this function is unknown, it is necessary to conduct a simulation experiment aimed at collecting statistical data, based on which it will be possible to perform approximation of the function $f$ in the neighborhood $\mathbf{Q}\left(\mathbf{x}^{k}, \delta\right)=\left\{\mathbf{x} \in \mathbb{R}^{S}:\left\|\mathbf{x}-\mathbf{x}^{k}\right\|<\delta\right\}$ of the point $\mathbf{x}^{k}$ (3), where $\delta=S$. The approximation may be performed on the basis of the regression analysis method.

\subsection{Local linear approximation of the criterion function}

Let us assume that the probabilistic model for the process of changing the number of documents on the shelf $s$ is a random variable $\xi_{s}: \Omega \rightarrow \mathbb{R}$, for $s \in \mathcal{S}$. The value of $x_{s}$ is interpreted as a realization of the random variable $\xi_{s}$, i.e. $\xi_{s}=x_{s}$. Therefore, the value of vector $\mathbf{x}=\left[x_{1}, x_{2}, \ldots, x_{S}\right]^{T}$ is interpreted as a realization of the multi-dimensional random variable $\boldsymbol{\xi}=\left[\xi_{1}, \xi_{2}, \ldots, \xi_{S}\right]^{T}$, i.e. $\xi=\mathbf{x}$. Let us further assume that the probabilistic model of the process of changing the length of time intervals of archiving the information about the RFIDtagged documents from set $\mathcal{D}$ is the random variable $\psi: \Omega \rightarrow \mathbb{R}$. The value of $y$ is interpreted as a realization of this random variable, i.e. $\psi=y$.

Let us assume that the stochastic model of influence of independent random variables $\xi_{1}, \xi_{2}, \ldots, \xi_{S}$ on the dependent random variable $\psi$ is the following equation

$$
\psi=\eta\left(\xi_{1}, \xi_{2}, \ldots, \xi_{S}\right)+\varepsilon,
$$

where: $\quad \eta: \mathbb{R}^{S} \rightarrow \mathbb{R} \quad$ is the response surface, $\varepsilon \sim W N\left(0, \sigma^{2}\right)$. The regression analysis method is often used to approximate the function $\eta$.

Theorem 1 (regression). If $\boldsymbol{\xi}$ and $\psi$ are random variables defined in the probabilistic space $(\Omega, \mathcal{F}, P)$, whereas $\mathrm{E}(\psi)<\infty$, and if $\mathrm{f}: \mathbb{R}^{S} \rightarrow \mathbb{R}$ is the measurable function, a mean-square error of the model, i.e.

$$
\mathrm{Q}(\mathrm{f})=\mathrm{E}\left\{[\psi-\mathrm{f}(\boldsymbol{\xi})]^{2}\right\},
$$

assumes the smallest value for the function $\mathrm{f}$ by the formula:

$$
\hat{\mathrm{f}}(\mathbf{x})=\mathrm{E}(\psi \mid\{\boldsymbol{\xi}=\mathbf{x}\}) .
$$

The function $\hat{\mathrm{f}}$ is called the regression model of the random variable $\psi$ with respect to the random variable $\xi$.

Let us assume that the unknown function $\eta$ belongs to the class of functions $\mathrm{H}$ including all linear regression functions with the vector parameters $\boldsymbol{\beta} \in \mathbb{R}^{P \times 1}$. It means that the response surface meets the equation $\mathrm{E}(\psi)=\eta(\mathbf{x}, \boldsymbol{\beta})$. The main idea of the response surface methodology [16] consists in the planning of experiments in such a manner that the mean-square error of the model with the vector parameter $\boldsymbol{\beta}$ is minimized.

Let us assume that in the neighborhood $\mathbf{Q}\left(\mathbf{x}^{k}, \delta\right)$ of the point $\mathbf{x}^{k}$ (3), the local model of the response surface is the linear regression function in the form of

$$
\mathrm{f}^{k}(\mathbf{x})=\mathbf{z}^{T} \boldsymbol{\beta}_{k}, \quad \mathbf{x} \in \mathbf{Q}\left(\mathbf{x}^{k}, \delta\right),
$$

where: $\mathbf{z}=\left[1, \mathbf{x}^{T}\right]^{T}, \boldsymbol{\beta}_{k}=\left[\beta_{k 0}, \beta_{k 1}, \ldots, \beta_{k S}\right]^{T}$ is vector of unknown parameters of the function $\mathrm{f}^{k}$. The estimation of the model $\mathrm{f}^{k}$ is based on the estimation of the vector 
$\boldsymbol{\beta}_{k}$. For that purpose, it is essential to come up with a plan of the simulation experiments allowing to collect the statistical data, based on which the estimation of this vector will be possible.

Let

$$
P_{k}=\left(\begin{array}{cccc}
\mathbf{z}_{1} & \mathbf{z}_{2} & \cdots & \mathbf{z}_{N} \\
p_{k 1} & p_{k 2} & \cdots & p_{k N}
\end{array}\right), \quad \mathbf{x} \in \mathbf{Q}\left(\mathbf{x}^{k}, \delta\right),
$$

be a normalized discrete plan of an experiment, where: $\mathbf{z}_{1}, \ldots, \mathbf{z}_{N}$ are focal points of the plan, in which the realizations of the random variable $\psi$ are observed for the fixed realizations of the multi-dimensional random variable $\boldsymbol{\xi}$, i.e. $\boldsymbol{\xi}_{\omega_{n}}=\mathbf{x}_{n}$, for $n=1, \ldots, N ; p_{k 1}, \ldots, p_{k N}$ are weights selected in such a manner that $\sum_{n=1}^{N} p_{k n}=1$. Let $\mathcal{N}=\{1, \ldots, N\}$ be a set of numbers of the focal points of the plan $P_{k}$. Let us assume that in the focal points, influence of the realizations of the random variable $\xi$ on the realizations of the random variable $\psi$ is described by the equations in the form of

$$
y_{n}=\mathrm{f}^{k}\left(\mathbf{x}_{n}\right)+\varepsilon_{n}=\mathbf{z}_{n}^{T} \boldsymbol{\beta}_{k}+\varepsilon_{n}, \quad n \in \mathcal{N},
$$

where: $y_{n}$ is the observed length of time intervals of archiving the information about the RFID-tagged documents from set $\mathcal{D}$ located on the shelves of the RFID cabinet in a manner determined by the value of vector $\mathbf{x}_{n}, \mathbf{x}_{n} \in \mathbf{Q}\left(\mathbf{x}^{k}, \delta\right) ; \mathbf{z}_{n}=\left[1, \mathbf{x}_{n}^{T}\right]^{T} ; \varepsilon_{n} \sim W N\left(0, \sigma^{2}\right)$.

We are looking for the linear regression model of mapping $\hat{\mathrm{f}}^{k}(6)$ in the form of

$$
\hat{y}_{n}=\hat{\mathrm{f}}^{k}\left(\mathbf{x}_{n}\right)=\mathbf{z}_{n}^{T} \mathbf{b}_{k}, \quad n \in \mathcal{N},
$$

where $\mathbf{b}_{k}=\left[b_{k 0}, b_{k 1}, \ldots, b_{k S}\right]^{T}$.

Let us rewrite equations (6) in the following matrix form:

$$
\mathbf{y}=\mathbf{Z} \boldsymbol{\beta}_{k}+\boldsymbol{\varepsilon},
$$

where: $\quad \mathbf{y}=\left[y_{1}, \ldots, y_{N}\right]^{T} \in \mathbb{R}^{N \times 1}, \quad \mathbf{Z}=\left[\mathbf{z}_{1}^{T}, \mathbf{z}_{2}^{T}, \ldots, \mathbf{z}_{N}^{T}\right]^{T}$, $\boldsymbol{\varepsilon}=\left[\varepsilon_{1}, \ldots, \varepsilon_{N}\right]^{T} \in \mathbb{R}^{N \times 1}$.

On the other hand, the equations (7) will have the following matrix form:

$$
\hat{\mathbf{y}}=\mathbf{Z} \mathbf{b}_{k},
$$

where: $\hat{\mathbf{y}}=\left[\hat{y}_{1}, \ldots, \hat{y}_{N}\right]^{T} \in \mathbb{R}^{N \times 1}$. Matrix $\mathbf{Z}$ is called the plan of an experiment.

The problem of estimating the model $\hat{\mathrm{f}}^{k}$ reduces to the issue of estimation of vector $\hat{\mathbf{b}}_{k}$ that minimizes the sum of squared errors of the model $\mathbf{e}=\mathbf{y}-\mathbf{Z} \mathbf{b}_{k}$.

This issue can be formulated as the optimization problem in the form of

$$
\mathrm{q}\left(\hat{\mathbf{b}}_{k}\right)=\min _{\mathbf{b}_{k} \in \mathbb{R}^{(S+1)}}\left[\mathrm{q}\left(\mathbf{b}_{k}\right)=\mathbf{e}^{T} \mathbf{e}\right] .
$$

Problem (8) is a linear squares issue [9-11]. Let us write the minimized sum of squared errors in the form of

$$
\begin{aligned}
\mathrm{q}\left(\mathbf{b}_{k}\right) & =\left(\mathbf{y}-\mathbf{Z} \mathbf{b}_{k}\right)^{T}\left(\mathbf{y}-\mathbf{Z} \mathbf{b}_{k}\right) \\
& =\mathbf{y}^{T} \mathbf{y}-\mathbf{y}^{T} \mathbf{Z} \mathbf{b}_{k}-\mathbf{b}_{k}^{T} \mathbf{Z}^{T} \mathbf{y}+\mathbf{b}_{k}^{T} \mathbf{Z}^{T} \mathbf{Z} \mathbf{b}_{k} \\
& =\mathbf{y}^{T} \mathbf{y}-2 \mathbf{b}_{k}^{T} \mathbf{Z}^{T} \mathbf{y}+\mathbf{b}_{k}^{T} \mathbf{Z}^{T} \mathbf{Z} \mathbf{b}_{k} .
\end{aligned}
$$

When we differentiate function $\mathrm{q}$ with respect to the vector $\mathbf{b}_{k}$, we obtain:

$$
\frac{\partial \mathrm{q}\left(\mathbf{b}_{k}\right)}{\partial \mathbf{b}_{k}}=-2 \mathbf{Z}^{T} \mathbf{y}+2 \mathbf{Z}^{T} \mathbf{Z} \mathbf{b}_{k} .
$$

According to the above formula, we obtain the system of the normal equation:

$$
\mathbf{Z}^{T} \mathbf{Z} \mathbf{b}_{k}=\mathbf{Z}^{T} \mathbf{y}
$$

If the matrix $\mathbf{Z}^{T} \mathbf{Z}$ is not singular, then the solution of problem (8) is the estimator in the form of

$$
\hat{\mathbf{b}}_{k}=\left(\mathbf{Z}^{T} \mathbf{Z}\right)^{-1} \mathbf{Z}^{T} \mathbf{y} \text {. }
$$

To facilitate the estimation of parameter vector $\hat{\mathbf{b}}_{k}(9)$, the complete two-level plan of experiments are often used [13]. According to this method, it is assumed that the number of focal points $N$ of plan $P_{k}$ (5) is $N=2^{S}$, and the components of the plan of experiment assume the values from the set $\{-1,1\}$. Let $\mathbf{U}=\left[u_{i j}\right]_{N \times(S+1)}$ be the plan of experiment, whose components $u_{i j}, i=1, \ldots, N$, $j=1, \ldots, S+1$, assume the values from this set. Figure 1 presents matrix $\mathbf{U}$ for $S=3$.

$$
\mathrm{U}=\left(\begin{array}{rrrr}
1 & -1 & -1 & -1 \\
1 & 1 & -1 & -1 \\
1 & -1 & 1 & -1 \\
1 & 1 & 1 & -1 \\
1 & -1 & -1 & 1 \\
1 & 1 & -1 & 1 \\
1 & -1 & 1 & 1 \\
1 & 1 & 1 & 1
\end{array}\right)
$$

Figure 1. Model plan of experiment

For plan $\mathbf{U}$, the equivalent of the estimator $\hat{\mathbf{b}}_{k}(9)$ is the vector $\hat{\mathbf{a}}_{k}$ in the form of

$$
\hat{\mathbf{a}}_{k}=\left(\mathbf{U}^{T} \mathbf{U}\right)^{-1} \mathbf{U}^{T} \mathbf{y}
$$

where: $\quad \hat{\mathbf{a}}_{k}=\left[\hat{a}_{k 0}, \hat{a}_{k 1}, \ldots, \hat{a}_{k S}\right]^{T}$, and the components $\hat{a}_{k 1}, \hat{a}_{k 2}, \ldots, \hat{a}_{k S}$ of this vector are equal to appropriate components of vector $\hat{\mathbf{b}}_{k}$, i.e. $\hat{a}_{k s}=\hat{b}_{k s}$, for $s=1, \ldots, S$. Since the covariance matrix $\left(\mathbf{U}^{T} \mathbf{U}\right)^{-1}$ is:

$$
\left(\mathbf{U}^{T} \mathbf{U}\right)^{-1}=\frac{1}{N} \mathbf{I}_{S},
$$

the estimator (8) has the following final form 


$$
\hat{\mathbf{a}}_{k}=\frac{1}{N} \mathbf{U}^{T} \mathbf{y},
$$

where $\mathbf{I}_{S}$ is an $S \times S$ identity matrix.

\subsection{Solution of the optimization problem}

Let us write vector $\hat{\mathbf{a}}_{k}=\left[\hat{a}_{k 0}, \hat{a}_{k 1}, \ldots, \hat{a}_{k S}\right]^{T}$ (11) in the form of $\hat{\mathbf{a}}_{k}=\left[\hat{a}_{k 0}, \hat{\mathbf{c}}_{k}^{T}\right]^{T}$, where $\hat{\mathbf{c}}_{k}=\left[\hat{a}_{k 1}, \ldots, \hat{a}_{k S}\right]^{T}$. As can be seen, the gradient of function $\hat{f}^{k}$ (6) has, in the point $\mathbf{x}^{k}$, the following form

$$
\nabla \hat{\mathrm{f}}^{k}\left(\mathbf{x}^{k}\right)=\hat{\mathbf{c}}_{k} .
$$

Applying algorithm (3) for solving problem (1) needs to determine a set of constraints active at the point $\mathbf{x}^{k}$ which is defined by a set of their indices

$$
\mathcal{A}\left(\mathbf{x}^{k}\right)=\left\{i \in\{1,2, \ldots, S+1\}: \mathbf{e}_{i}^{T} \mathbf{x}^{k}=n_{i}\right\},
$$

where: $\mathbf{e}_{i}=\left\{\begin{array}{ll}\mathbf{a}_{i}, & \text { for } 1 \leq i \leq S, \\ \mathbf{1}, & \text { for } i=S+1,\end{array}, n_{i}= \begin{cases}m_{i}, & \text { for } 1 \leq i \leq S, \\ D, & \text { for } i=S+1 .\end{cases}\right.$

On the basis of set $\mathcal{A}\left(\mathbf{x}^{k}\right)$ defines a matrix $\tilde{\mathbf{A}}_{k}$, whose rows are vectors $\mathbf{e}_{i}$, for $i \in \mathcal{A}\left(\mathbf{x}^{k}\right)$. Next is created a matrix of constraints $\mathbf{A}_{k}$ significantly active in the point $\mathbf{x}^{k}$. This matrix is formed in such a way that linearly dependent rows are removed from matrix $\tilde{\mathbf{A}}_{k}$.

Direction $\mathbf{d}^{k}$ (3) is calculated according to the following formula:

$$
\mathbf{d}^{k}=\left\{\begin{array}{cc}
-\mathbf{w}^{k}+\beta_{k} \mathbf{d}^{k-1}, & \text { for } k>0, \\
-\mathbf{w}^{k}, & \text { for } k=0,
\end{array}\right.
$$

where:

- $\beta_{k}$ is a coefficient determined by the formula,

$$
\beta_{k}=\left\{\begin{array}{cc}
0, & \text { for } k=0, \\
\frac{\left(\mathbf{w}^{k}\right)^{T}\left(\mathbf{w}^{k}-\mathbf{w}^{k-1}\right)}{\left\|\mathbf{w}^{k-1}\right\|^{2}}, & \text { for } k>0,
\end{array}\right.
$$

- $\mathbf{w}^{k}$ is a vector of the form,

$$
\mathbf{w}^{k}=\left\{\begin{array}{cc}
\nabla \hat{\mathrm{f}}^{k}\left(\mathbf{x}^{k}\right), & \text { for } k=0, \\
\mathbf{P}_{k} \nabla \hat{\mathrm{f}}^{k}\left(\mathbf{x}^{k}\right), & \text { for } k>0,
\end{array}\right.
$$

- $\mathbf{P}_{k}$ is an orthogonal projection matrix in the form of

$$
\mathbf{P}_{k}=\mathbf{I}_{S}-\mathbf{A}_{k}^{T}\left(\mathbf{A}_{k} \mathbf{A}_{k}^{T}\right)^{-1} \mathbf{A}_{k} .
$$

Reapplication of the above procedure, consisting in applying the substitution $\mathbf{d}^{k}=\mathbf{w}^{k}$, is performed in the following cases:
- $\mathbf{d}^{k}$ is not a descending direction,

- when matrix $\mathbf{A}_{k}$ is changed, i.e. when $\mathbf{A}_{k} \neq \mathbf{A}_{k-1}$,

- when from the last round it was made of $S-\operatorname{rank}\left(\mathbf{A}_{k}\right)$ iterations.

The basic criterion for terminating the above procedure is when the norm of vector $\mathbf{w}^{k}$ reaches a zero value with accuracy $\varepsilon>0$, i.e. $\left\|\mathbf{w}^{k}\right\| \leq \varepsilon$.

Example 1. Let us consider the issue of optimization the placement of the RFID-tagged documents in the cabinet equipped with $S=4$ document shelves. The numbers of free spaces on particular shelves are: $m_{1}=100, m_{2}=10$, $m_{3}=5, m_{4}=40$. Let us assume that we need to place $D=100$ RFID-tagged documents in the cabinet. On the basis of the simulation experiments and by applying the optimization algorithm described in section 3 , the following solution is reached:

$$
\hat{\mathbf{x}}=[45,10,5,40]^{T} .
$$

\section{Summary and direction of further work}

The optimization approach to the issue of placing the RFID-tagged documents ha been discussed in the work. Since the form of the criterion function is not explicitly known, to solve the optimization problem, the computer simulation method has been applied. On the basis of such data, the local linear approximation of the criterion function has been made by using the regression analysis method. The method required, among other things, to prepare the plans of the simulation experiments. For this, the complete two-level designs have been used.

Further work should focus on important from a practical point of view, the issues of grouping documents and their movement between different RFID cabinets. To solve these problems, the method of the facility location problem may be applied [17-19].

\section{References}

1. D. Hunt, A. Puglia, M. Puglia, RFID: A Guide to Radio Frequency Identification (Wiley-Blackwell, 2007)

2. C. Heinrich, RFID and Beyond (Wiley Publishing, Indianapolis, 2005)

3. W. I. Zangwill, Nonlinear Programming: A Unified Approach (Englewood Cliffs: Prentice-Hall, 1969)

4. E. Polak, Computational Methods in Optimization: A Unified Approach (Academic Press, 1971).

5. G. S. Fishman, Discrete-Event Simulation: Modeling, Programming, and Analysis (Springer, 2001)

6. G. A. Wainer, P. J. Mosterman (Eds.), DiscreteEvent Modeling and Simulation: Theory and Applications, (CRC Press, 2010)

7. P. C. Kleijnen, Design and Analysis of Simulation Experiments (Springer, 2010) 
8. B. Zeigler, H.S. Sarjoughian, Guide to Modeling and Simulation of Systems of Systems (Springer, 2012)

9. G.E.P. Box, G.M. Jenkins, G.C. Reinsel, Time Series Analysis. Forecasting and Control (John Willey and Sons, 2008)

10. J. Fan, Q. Yao, Nonlinear Time Series. Nonparametric and Parametric Methods (SpringerVerlag Inc., 2003)

11. L. Ljung, System Identification. Theory for the User, (PTR Prentice Hall, 1999)

12. F. Pukelsheim, Optimal Design of Experiments (Society for Industrial \& Applied Mathematics, 2006)

13. M. Morris, Design of Experiments: An Introduction Based on Linear Models (Chapman \& Hall/CRC Texts in Statistical Science, 2010)
14. M. A. Bhatti, Practical Optimization Methods with Mathematica Applications (Springer-Verlag Inc., 2000)

15. P. H. Calamai, J. J.Moré, Projected Gradient Methods for Linearly Constrained Problems, (Mathematical Programming, 39, 1987, pp. 93-116)

16. G. E. P. Box, R. D. Norman, Response Surfaces, Mixtures, and Ridge Analyses (Wiley Series in Probability and Statistics. Wiley-Blackwell, 2007)

17. J. A. Eiman, Models and Algorithms for the Capacitated Facility Location Problem: Basics, Concepts, Methods (LAP LAMBERT Academic Publishing, 2014)

18. Ch. N. Vijeyamurthy, Genetic Algorithm for Facility Location Problem (LAP LAMBERT Academic Publishing, 2013)

19. R. Martin, Facility Location and Related Problems (Südwestdeutscher Verlag für Hochschulschriften, (2009) 